\title{
Anomalous levitation and annihilation in Floquet topological insulators
}

\author{
Hui Liu, ${ }^{1}$ Ion Cosma Fulga, ${ }^{1}$ and János K. Asbóth ${ }^{2,3}$ \\ ${ }^{1}$ IFW Dresden and Würzburg-Dresden Cluster of Excellence ct.qmat, Helmholtzstrasse 20, 01069 Dresden, Germany \\ ${ }^{2}$ Institute for Solid State Physics and Optics, Wigner Research Centre for Physics, P.O. Box 49, H-1525 Budapest, Hungary \\ ${ }^{3}$ Department of Theoretical Physics, Budapest University of Technology and Economics, Budapest, Hungary
}

(Received 10 March 2020; accepted 14 May 2020; published 4 June 2020)

\begin{abstract}
Anderson localization in two-dimensional topological insulators takes place via the so-called levitation and pair annihilation process. As disorder is increased, extended bulk states carrying opposite topological invariants move towards each other in energy, reducing the size of the topological gap, eventually meeting and localizing. This results in a topologically trivial Anderson insulator. Here, we introduce the anomalous levitation and pair annihilation, a process unique to periodically driven, or Floquet, systems. Due to the periodicity of the quasienergy spectrum, we find it is possible for the topological gap to increase as a function of disorder strength. Thus, after all bulk states have localized, the system remains topologically nontrivial, forming an anomalous Floquet-Anderson insulator (AFAI) phase. We show a concrete example for this process, adding disorder via on-site potential "kicks" to a Chern insulator model. By changing the period between kicks, we can tune which type of (conventional or anomalous) levitation and annihilation occurs in the system. We expect our results to be applicable to generic Floquet topological systems and to provide an accessible way to realize AFAIs experimentally, without the need for multistep driving schemes.
\end{abstract}

DOI: 10.1103/PhysRevResearch.2.022048

Introduction. In fully coherent systems, disorder leads to a loss of metallic conduction and a transition to a localized state: the Anderson insulator (AI) [1,2]. In three dimensions, this is a gradual process. For small disorder, each energy band is split by so-called mobility edges to a middle part with extended states and outer parts with localized states, whose localization length diverges at the mobility edges. As disorder is increased, the central, extended part of each band shrinks, and eventually the two mobility edges meet and the bands become localized. In contrast, in generic one- and two-dimensional systems, already an arbitrarily weak disorder is enough to localize all bulk states.

Shortly after the discovery of the quantum Hall effect [3], it was realized that two-dimensional Chern insulators also require a finite amount of disorder to localize, but through a different type of transition [4]. In bands with a nonzero Chern number, although almost all bulk eigenstates can be (and are) exponentially localized, the localization length diverges at isolated energies: Extended states "carry the Chern number" [5-7]. As found by Laughlin [4], the extended bulk states carrying opposite Chern numbers "levitate" towards each other in energy when disorder is gradually increased, and eventually "annihilate" pairwise, so Anderson localization sets in [8]. Since the topological edge states only occur within the mobility gap between the extended bulk states, the pair annihilation leads to a topologically trivial system.

Published by the American Physical Society under the terms of the Creative Commons Attribution 4.0 International license. Further distribution of this work must maintain attribution to the author(s) and the published article's title, journal citation, and DOI.
In the last decade, it was found that robust extended edge states and complete bulk Anderson localization can coexist in periodically driven systems, so-called anomalous FloquetAnderson insulators (AFAIs) [9-11]. Even if all quasienergy bands have zero Chern numbers, Floquet insulators can have topologically protected chiral edge states, which wind in quasienergy [11-20]. Since the bands are trivial, arbitrarily weak disorder leads to a fully localized bulk, while leaving the chiral edge states extended [9-11]: There is no levitation and annihilation in such anomalous Floquet topological insulators.

In this Rapid Communication, we revisit Laughlin's result on Anderson localization in the context of Floquet Chern insulators [21-33]. The quasienergy bands of these systems carry Chern numbers, and hence we expect a levitation-andannihilation scenario [34]. However, even in the simplest two-band models, there are two different ways in which extended states carrying opposite Chern numbers can meet and annihilate. Due to the periodic spectrum, the extended states can levitate towards each other by reducing the size of the topological gap (the conventional scenario) or by increasing it instead (see Fig. 1). Thus, disorder can induce a transition from a Floquet Chern insulator not only to an AI, but also to an AFAI. We show this in the following using a toy model for a Floquet Chern insulator, in which both scenarios of levitation and annihilation happen, and find a simple rule of thumb for when to expect either scenario.

System. We consider a tight-binding model on a square lattice, with Hamiltonian [36]

$$
\begin{aligned}
\hat{H}_{0}= & v_{1}(1+i) \sum_{\mathbf{r}}\left(\left|A_{\mathbf{r}}\right\rangle\left\langle B_{\mathbf{r}}|+| A_{\mathbf{r}+\mathbf{a}_{x}+\mathbf{a}_{y}}\right\rangle\left\langle B_{\mathbf{r}}|+| B_{\mathbf{r}}\right\rangle\left\langle A_{\mathbf{r}+\mathbf{a}_{x}}\right|\right. \\
& \left.+\left|B_{\mathbf{r}}\right\rangle\left\langle A_{\mathbf{r}+\mathbf{a}_{y}}\right|\right)+v \sum_{\mathbf{r}}\left(\left|A_{\mathbf{r}}\right\rangle\left\langle A_{\mathbf{r}+\mathbf{a}_{x}}|-| A_{\mathbf{r}}\right\rangle\left\langle A_{\mathbf{r}+\mathbf{a}_{y}}\right|\right. \\
& \left.+\left|B_{\mathbf{r}}\right\rangle\left\langle B_{\mathbf{r}+\mathbf{a}_{y}}|-| B_{\mathbf{r}}\right\rangle\left\langle B_{\mathbf{r}-\mathbf{a}_{x}}\right|\right)+ \text { H.c., }
\end{aligned}
$$




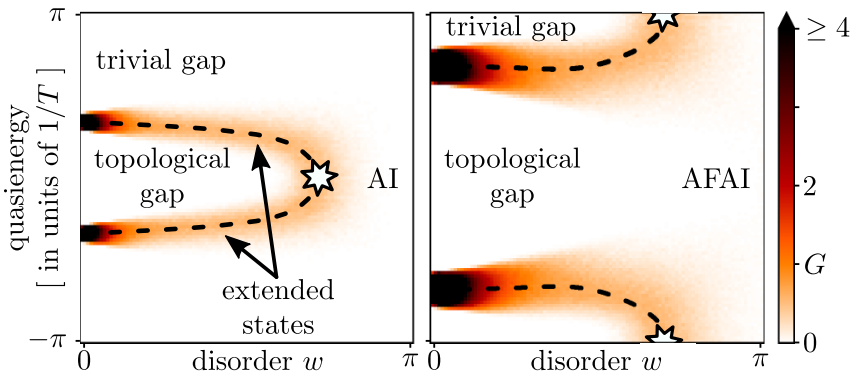

FIG. 1. When increasing disorder, the extended states separating topological and trivial quasienergy gaps levitate towards each other, eventually annihilating pairwise (white star). The conventional form of this process leads to the elimination of the topological gap, resulting in a trivial Anderson insulator (AI, left panel). The anomalous levitation and pair annihilation, in which the trivial gap shrinks, leaves behind an anomalous Floquet-Anderson insulator (AFAI, right panel). The background colors are numerically obtained by computing the transmission, as explained in the main text [35].

where $\left|A_{\mathbf{r}}\right\rangle$ and $\left|B_{\mathbf{r}}\right\rangle$ denote sites on the $A$ and $B$ sublattice in the unit cell (see Fig. 2) with coordinates $\mathbf{r}=N_{x} \mathbf{a}_{x}+N_{y} \mathbf{a}_{y}$, with $N_{x}, N_{y} \in \mathbb{Z}$. We measure energy in units of $v$, time in units of $1 / v$ ( $\hbar=1$ throughout $)$, and distance along $x$ and $y$ in units of $\left|a_{x}\right|$ and $\left|a_{y}\right|$. The two energy bands are symmetric around $E=0$ because $\operatorname{tr} \hat{H}_{0}(k)=0$. For most of this Rapid Communication we will use $v_{1}=v$, where the bands have Chern numbers \pm 1 , and thus the gap separating them is topological, i.e., hosts one branch of chiral edge states. Here, as Fig. 2(b) shows, the bands are relatively flat: Their bandwidths $1.17 v$ are much smaller than the band gap $\Delta=5.66 v$.

We add disorder to the hopping model in the form of periodic on-site potential kicks,

$$
\hat{H}(t)=\hat{H}_{0}+w \hat{H}_{\text {dis }} \sum_{n \in \mathbb{Z}} \delta(t-n T) .
$$
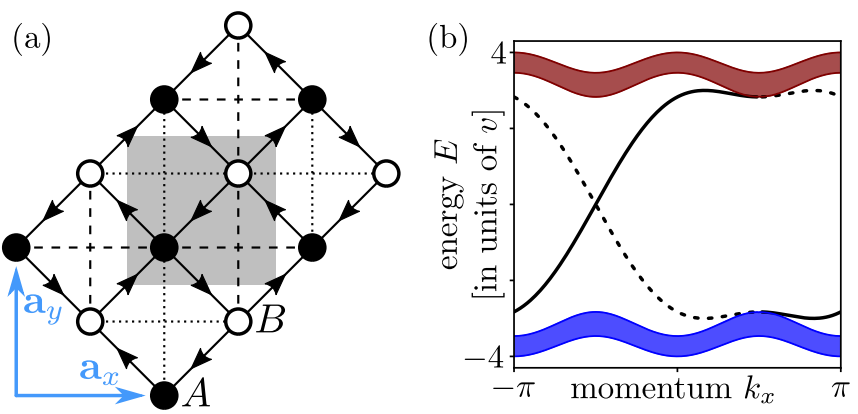

FIG. 2. (a) The model Hamiltonian: One unit cell (gray square) contains two sites, belonging to the $A$ and $B$ sublattice (solid and open circle, respectively). Bravais vectors $\mathbf{a}_{x, y}$ are indicated as blue arrows. Nearest-neighbor hopping amplitudes along and against the arrows are $v_{1}(1+i)$ and $v_{1}(1-i)$, respectively. Next-nearest-neighbor hopping amplitudes are $+v$ along the dashed lines and $-v$ along the dotted lines. (b) Dispersion relation computed for $v_{1}=v$ in a ribbon geometry, infinite along $\mathbf{a}_{x}$, and consisting of 20 unit cells in the $\mathbf{a}_{y}$ direction. Top and bottom edge modes are shown as dashed and solid black lines, respectively.
The time period $T$ separates the kicks, which have a strength $w \in \mathbb{R}$ and are spatially random,

$$
\hat{H}_{\text {dis }}=\sum_{r}\left(\xi_{\mathbf{r}, A}\left|A_{\mathbf{r}}\right\rangle\left\langle A_{\mathbf{r}}\left|+\xi_{\mathbf{r}, B}\right| B_{\mathbf{r}}\right\rangle\left\langle B_{\mathbf{r}}\right|\right),
$$

with $\xi_{r, A / B}$ random numbers drawn independently for each lattice site, uniformly distributed with $-1 \leqslant \xi \leqslant 1$. Note that the delta function in Eq. (2) has units of inverse time, or energy, such that both $w$ and $\xi$ are dimensionless.

The Floquet spectrum is the spectrum of the Floquet operator $\hat{\mathcal{F}}$, the time evolution operator of one period,

$$
\hat{\mathcal{F}}=\mathcal{T} e^{-i \int_{0}^{T} \hat{H}\left(t^{\prime}\right) d t^{\prime}}=e^{-i w \hat{H}_{\mathrm{dis}}} e^{-i \hat{H}_{0} T},
$$

where $\mathcal{T}$ denotes time ordering. Eigenstates of $\hat{\mathcal{F}}$-the Floquet eigenstates-pick up phase factors of $e^{-i \varepsilon T}$ during each period, where the quasienergy $\varepsilon$ takes values in $[-\pi / T, \pi / T]$ - the Floquet zone, in analogy with the Brillouin zone. In the limit of maximal disorder, $w=\pi$, the kicks randomize quasienergy completely, meaning that all disorder-averaged properties of the model are independent of quasienergy.

As a first step to understanding the effects of the periodic kicks, we take the limit of vanishing kick strength $w \rightarrow 0$, similarly to the way lattice effects are treated in the nearlyfree-electron model of crystalline solids. This amounts to time evolution using the static Hamiltonian $\hat{H}_{0}$ but calculating the effects only at integer multiples of a time period $T$. In the absence of kicks, the time period $T$ does not change any of the physical properties of the system, only the type of information we can extract from it: Any eigenstate of $\hat{H}_{0}$, with energy $E$, is also an eigenstate of $\hat{\mathcal{F}}$. The corresponding quasienergy is $\varepsilon=E$, projected into the first Floquet zone, i.e., $\varepsilon=[(E T+\pi) \bmod (2 \pi)-\pi] / T$. As in the nearlyfree-electron model, we will use a repeated Floquet zone description here, and for simplicity sometimes argue using the "phase per period" $\varepsilon T$, which is the same as the quasienergy $\varepsilon$ measured in units of $1 / T$.

Topology. Even in the limit of vanishing kick strength, $w \rightarrow 0$, the time period $T$ can be used to tune the topological invariants of the system, the winding numbers $\mathcal{W}$ [13] of the quasienergy gaps. To see this, we follow the quasienergy bands in a repeated Floquet zone scheme, in Fig. 3. For $T<0.25 \pi / v$, all the energy spectrum of $\hat{H}_{0}$ fits in the first Floquet zone, including edge states. Thus the quasienergy spectrum consists of Floquet replicas of the lower and upper band, together with the edge states between them, and the gap around $\varepsilon=0$ is topological, whereas the gap at the Floquet zone boundary, $\varepsilon=\pi / T$, is trivial. As $T$ is increased, the gap at $\varepsilon=0$ grows relative to the gap at $\varepsilon=\pi / T$, and eventually overtakes it at a critical period time,

$$
T_{c}=\frac{\pi}{2 E_{1 / 2}}=\frac{\pi}{(4+2 \sqrt{2}) v} \approx \frac{0.15 \pi}{v},
$$

where $E_{1 / 2}$ is the band center, the average of the minimum and maximum energies of the upper band [37]. At around $T \approx 2 T_{c}$ the bands cross the Floquet zone boundaries: The $\varepsilon=\pi / T$ gap closes, and when it reopens, hosts edge states coming from the first as well as from the second Floquet zones. Thus, the winding number of this gap changes from 


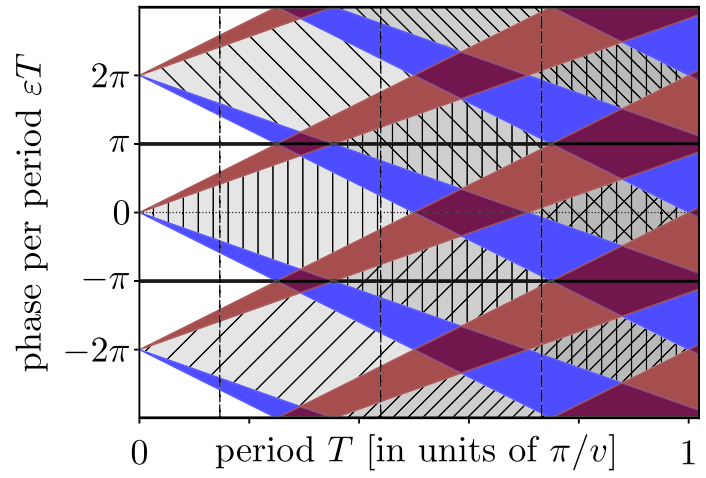

FIG. 3. Floquet spectrum of Eq. (4) with $v_{1}=v$ in the clean limit, $w=0$, in a repeated Floquet zone scheme. Tuning the time period $T$ has no physical effects here, as it only changes our description of the same physics, described by the constant Hamiltonian $\hat{H}_{0}$ - as in the first step of the nearly-free-electron approximation of crystalline solids. Hatching indicates the presence of edge states in a gap. Increasing the period $T$, bands cross over between Floquet zones, delimited by thick horizontal lines. This results in a sequence of topological phase transitions: Gaps are closed and reopened (even if in this undriven case no transitions between bands happen), with the number of edge states in them increasing by 2 each time [37]. Vertical dashed lines indicate periods for which the gaps are equal, $T=n T_{c}$ with $n=1,3,5$.

0 to 2 . Further increasing $T$ leads to a sequence of similar transitions at $T=2 n T_{c}$, at $\varepsilon=0$ for even $n$ and $\varepsilon=\pi / T$ for odd $n$.

Disorder. To investigate how disorder $w$ affects the system we calculate the two-terminal transmission $G$ using the KWANT code [37,38]. We consider a finite system of $L \times L$ unit cells, with either periodic or open boundary conditions in the $\mathbf{a}_{x}$ direction, and semi-infinite leads attached at the top and bottom, modeled as absorbing terminals at $N_{y}=1$ and $N_{y}=L$. The two-terminal scattering matrix $\hat{S}$ reads [39]

$$
\hat{S}(\varepsilon)=\hat{P}\left[1-e^{i \varepsilon} \hat{\mathcal{F}}\left(1-\hat{P}^{T} \hat{P}\right)\right]^{-1} e^{i \varepsilon} \hat{\mathcal{F}} \hat{P}^{T},
$$

where $\hat{P}$ is the projection operator onto the absorbing terminals. The total transmission $G(\varepsilon)$ can be extracted from the scattering matrix $S(\varepsilon)$,

$$
G=\operatorname{tr}\left(\mathfrak{t}^{\dagger} \mathfrak{t}\right), \quad S=\left(\begin{array}{ll}
\mathfrak{r} & \mathfrak{t}^{\prime} \\
\mathfrak{t} & \mathfrak{r}^{\prime}
\end{array}\right),
$$

where $\mathfrak{r}^{(\prime)}$ and $\mathfrak{t}^{(\prime)}$ are the blocks containing probability amplitudes for back-reflection, or transmission between the two terminals, respectively, whose dependence on $\varepsilon$ was suppressed for readability. With periodic boundary conditions and at maximal disorder, $w=\pi$, the transmission is quasienergy independent, such that a vanishing transmission at any value of $\varepsilon$ indicates total localization of all bulk states. Changing to open boundary conditions, for an AFAI phase with topological invariant $\mathcal{W}$, topologically protected edge states will appear, constitute completely open channels for transport, and contribute integer values to the total transmission, with $G(\varepsilon)=|\mathcal{W}|$ for all $\varepsilon$. Alternatively, the invariant $\mathcal{W}$ can be obtained as the winding of the determinant of the reflection part $\mathfrak{r}$ of the scattering matrix $[37,39,40]$.

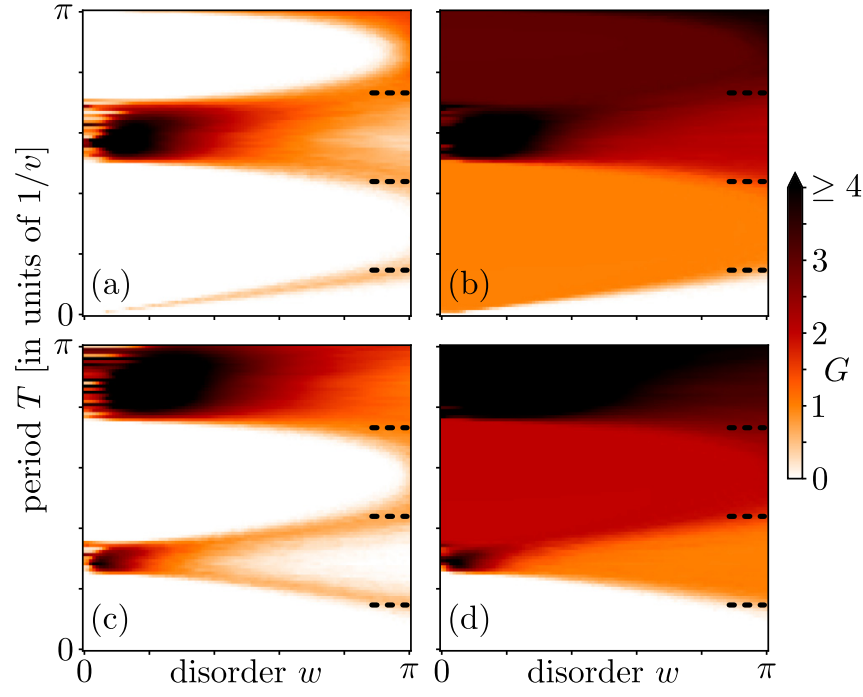

FIG. 4. Phase diagram of the transmission $G$ depending on disorder $w$ and period $T$, at quasienergy (a), (b) $\varepsilon=0$ and (c), (d) $\varepsilon=\pi$. The boundary conditions along $x$ are periodic in (a) and (c), and open in (b) and (d). Transmissions are calculated for a system size of $20 \times 20$, with $v_{1}=v$, and by averaging over 50 disorder realizations. Dashed lines show the analytically predicted phase transition points, as described in the main text.

The calculated values of the transmission (see Fig. 4) show that, depending on the period $T$, increasing disorder strength can lead to a transition to an AI or an AFAI, with either the usual or the anomalous levitation-and-annihilation scenarios of Fig. 1. For $T \ll 0.25 \pi / v$, we find the first scenario: In Fig. 4(a) the mobility gap at $\varepsilon=0$ closes and reopens as two extended states carrying the Chern numbers meet and annihilate, while the winding number of this gap changes from 1 to 0 , as evidenced in Fig. 4(b). We show an example in more detail, for $T=0.1 \pi / v$, in Fig. 1. For a range of period times $(0.15 \pi / v<T<0.45 \pi / v)$, we find the second scenario, a transition to AFAI via an anomalous levitation and annihilation: In Fig. 4(c), the mobility gap closes and reopens at $\varepsilon=\pi / T$, and edge transmission indicates a winding number of 1 in the $w=\pi$ limit, in Figs. 4(b) and 4(d). An example, with $T=0.2 \pi / v$, is shown in Fig. 1. For longer drive periods we observe hints of transitions to AFAI phases with higher winding numbers: around $T \approx 0.5 \pi / v$, of $\mathcal{W}=$ 2 , and around $T=0.9 \pi / v$, possibly $\mathcal{W}=3$, although with substantial finite-size effects.

We find a simple rule of thumb to predict whether maximal disorder $(w=\pi)$ leads to an AI or an AFAI: The winding number $\mathcal{W}$ of the fully localized phase at $w=\pi$ is given by the winding number of the dominant gap in the case without disorder, $w=0$. We thus expect phase transitions between AI and AFAI to occur at $T=(2 m+1) T_{c}$, with $m \in \mathbb{N}$ and $T_{c}$ given by Eq. (5). This is already seen in the data of Fig. 4, where the dashed lines showing the expected transitions agree well with the data. However, it also holds in the more general case, with $v_{1} \neq v$ in the Hamiltonian of Eq. (1), as shown in Fig. 5. Here, we again find good agreement between the numerically obtained phase transitions and the condition $T=(2 m+1) T_{c}$, now with $T_{c}$ depending on 


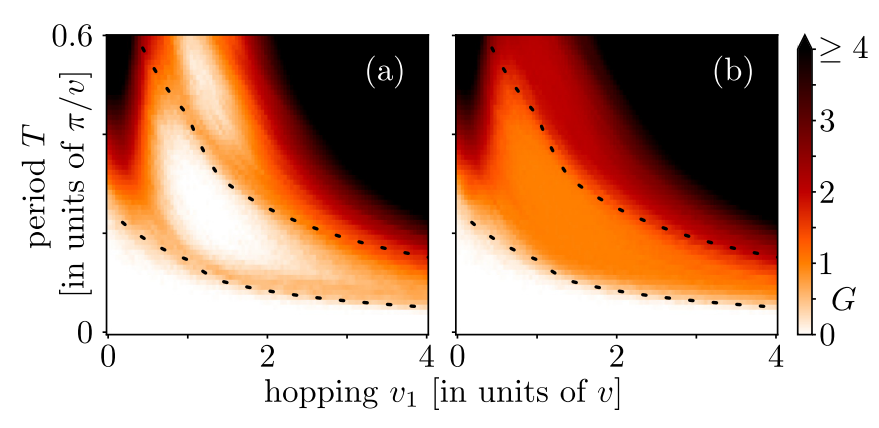

FIG. 5. Phase diagram of the transmission with maximal disorder, $w=\pi$. With (a) periodic boundary conditions, white regions of low transmission, separated by ridges, are the AI and AFAI phases. The phase boundaries are well approximated by the analytical predictions (dotted lines), where $w=0$ gaps have equal sizes. With (b) open boundary conditions, we can read off the topological invariants of the Anderson localized phases via the quantized value of transmission. The system size is $20 \times 20$, and each point is obtained by averaging over 50 disorder realizations.

$v_{1}$ in a piecewise linear fashion (see Supplemental Material [37]).

Conclusion and discussion. We have shown that one can realize the AFAI, i.e., full Anderson localization in the bulk and topologically protected edge states, by adding disorder in the form of on-site potential kicks to a Chern insulator. The transition to the AFAI phase takes place via an anomalous form of the levitation and annihilation of extended states carrying the Chern numbers, different from previously studied cases $[9,41,42]$. The winding number of the fully disordered $w=\pi$ system is simply given by the winding number of the largest quasienergy gap at $w=0$. It would be interesting to explore whether this simple rule still holds in models with more than two bands. It would also be interesting to consider this process in different symmetry classes, where weak antilocalization can lead to metallic phases, in higherorder topological insulators [43-47], or in quantum walks $[48,49]$. Finally, we believe that our approach of using on-site potential "kicks" might offer an experimentally more viable route towards AFAI phases than the ones relying on more complicated, multistep driving protocols.

Acknowledgments. We thank Ulrike Nitzsche for technical assistance, and Tibor Rakovszky for discussions. This work is supported by the Deutsche Forschungsgemeinschaft (DFG, German Research Foundation) through the WürzburgDresden Cluster of Excellence on Complexity and Topology in Quantum Matter - ct.qmat (EXC 2147, project-id 39085490). J.K.A. acknowledges support from the National Research, Development and Innovation Fund of Hungary within the Quantum Technology National Excellence Program (Project No. 2017-1.2.1-NKP-2017-00001), and FK 124723 and FK 132146.
[1] P. W. Anderson, Absence of diffusion in certain random lattices, Phys. Rev. 109, 1492 (1958).

[2] F. Evers and A. D. Mirlin, Anderson transitions, Rev. Mod. Phys. 80, 1355 (2008).

[3] K. v. Klitzing, G. Dorda, and M. Pepper, New Method for High-Accuracy Determination of the Fine-Structure Constant based on Quantized Hall Resistance, Phys. Rev. Lett. 45, 494 (1980).

[4] R. B. Laughlin, Levitation of Extended-State Bands in a Strong Magnetic Field, Phys. Rev. Lett. 52, 2304 (1984).

[5] B. I. Halperin, Quantized Hall conductance, current-carrying edge states, and the existence of extended states in a twodimensional disordered potential, Phys. Rev. B 25, 2185 (1982).

[6] D. J. Thouless, Wannier functions for magnetic sub-bands, J. Phys. C: Solid State Phys. 17, L325 (1984).

[7] T. Thonhauser and D. Vanderbilt, Insulator/Chern-insulator transition in the Haldane model, Phys. Rev. B 74, 235111 (2006).

[8] E. Prodan, Disordered topological insulators: A non-commutative geometry perspective, J. Phys. A: Math. Theor. 44, 113001 (2011).

[9] P. Titum, E. Berg, M. S. Rudner, G. Refael, and N. H. Lindner, Anomalous Floquet-Anderson Insulator as a Nonadiabatic Quantized Charge Pump, Phys. Rev. X 6, 021013 (2016).

[10] A. Kundu, M. Rudner, E. Berg, and N. H. Lindner, Quantized large-bias current in the anomalous Floquet-Anderson insulator, Phys. Rev. B 101, 041403(R) (2020).

[11] F. Nathan, M. S. Rudner, N. H. Lindner, E. Berg, and G. Refael, Quantized Magnetization Density in Periodically Driven Systems, Phys. Rev. Lett. 119, 186801 (2017).
[12] T. Kitagawa, E. Berg, M. Rudner, and E. Demler, Topological characterization of periodically driven quantum systems, Phys. Rev. B 82, 235114 (2010).

[13] M. S. Rudner, N. H. Lindner, E. Berg, and M. Levin, Anomalous Edge States and the Bulk-Edge Correspondence for Periodically Driven Two-Dimensional Systems, Phys. Rev. X 3, 031005 (2013).

[14] F. Nathan and M. S. Rudner, Topological singularities and the general classification of Floquet-Bloch systems, New J. Phys. 17, 125014 (2015).

[15] D. V. Else and C. Nayak, Classification of topological phases in periodically driven interacting systems, Phys. Rev. B 93, 201103(R) (2016).

[16] S. Mukherjee, A. Spracklen, M. Valiente, E. Andersson, P. Öhberg, N. Goldman, and R. R. Thomson, Experimental observation of anomalous topological edge modes in a slowly driven photonic lattice, Nat. Commun. 8, 13918 (2017).

[17] L. J. Maczewsky, J. M. Zeuner, S. Nolte, and A. Szameit, Observation of photonic anomalous Floquet topological insulators, Nat. Commun. 8, 13756 (2017).

[18] A. Quelle, C. Weitenberg, K. Sengstock, and C. M. Smith, Driving protocol for a Floquet topological phase without static counterpart, New J. Phys. 19, 113010 (2017).

[19] I. C. Fulga, M. Maksymenko, M. T. Rieder, N. H. Lindner, and E. Berg, Topology and localization of a periodically driven Kitaev model, Phys. Rev. B 99, 235408 (2019).

[20] K. Wintersperger, C. Braun, F. N. Ünal, A. Eckardt, M. D. Liberto, N. Goldman, I. Bloch, and M. Aidelsburger, Realization of anomalous Floquet topological phases with ultracold atoms, arXiv:2002.09840. 
[21] T. Oka and H. Aoki, Photovoltaic Hall effect in graphene, Phys. Rev. B 79, 081406(R) (2009).

[22] J.-i. Inoue and A. Tanaka, Photoinduced Transition between Conventional and Topological Insulators in Two-Dimensional Electronic Systems, Phys. Rev. Lett. 105, 017401 (2010).

[23] N. H. Lindner, G. Refael, and V. Galitski, Floquet topological insulator in semiconductor quantum wells, Nat. Phys. 7, 490 (2011).

[24] Z. Gu, H. A. Fertig, D. P. Arovas, and A. Auerbach, Floquet Spectrum and Transport through an Irradiated Graphene Ribbon, Phys. Rev. Lett. 107, 216601 (2011).

[25] T. Kitagawa, T. Oka, A. Brataas, L. Fu, and E. Demler, Transport properties of nonequilibrium systems under the application of light: Photoinduced quantum Hall insulators without Landau levels, Phys. Rev. B 84, 235108 (2011).

[26] Y. T. Katan and D. Podolsky, Modulated Floquet Topological Insulators, Phys. Rev. Lett. 110, 016802 (2013).

[27] T. Iadecola, D. Campbell, C. Chamon, C.-Y. Hou, R. Jackiw, S.-Y. Pi, and S. V. Kusminskiy, Materials Design from Nonequilibrium Steady States: Driven Graphene as a Tunable Semiconductor with Topological Properties, Phys. Rev. Lett. 110, 176603 (2013).

[28] J. Cayssol, B. Dóra, F. Simon, and R. Moessner, Floquet topological insulators, Phys. Status Solidi RRL 7, 101 (2013).

[29] N. Goldman and J. Dalibard, Periodically Driven Quantum Systems: Effective Hamiltonians and Engineered Gauge Fields, Phys. Rev. X 4, 031027 (2014).

[30] A. G. Grushin, Á. Gómez-León, and T. Neupert, Floquet Fractional Chern Insulators, Phys. Rev. Lett. 112, 156801 (2014).

[31] A. Kundu, H. A. Fertig, and B. Seradjeh, Effective Theory of Floquet Topological Transitions, Phys. Rev. Lett. 113, 236803 (2014).

[32] P. Titum, N. H. Lindner, M. C. Rechtsman, and G. Refael, Disorder-Induced Floquet Topological Insulators, Phys. Rev. Lett. 114, 056801 (2015).

[33] M. Bukov, L. D’Alessio, and A. Polkovnikov, Universal highfrequency behavior of periodically driven systems: From dynamical stabilization to Floquet engineering, Adv. Phys. 64, 139 (2015).

[34] S. Roy and G. J. Sreejith, Disordered Chern insulator with a two-step Floquet drive, Phys. Rev. B 94, 214203 (2016).

[35] The transmission $G$ is obtained for system sizes $60 \times 60$ and $v_{1}=v$ by averaging over 1000 disorder realizations. Left and right panels show results for $T=0.1 \pi / v$ and $0.2 \pi / v$, respectively.

[36] T. Neupert, L. Santos, C. Chamon, and C. Mudry, Fractional Quantum Hall States at Zero Magnetic Field, Phys. Rev. Lett. 106, 236804 (2011).

[37] See Supplemental Material at http://link.aps.org/supplemental/ 10.1103/PhysRevResearch.2.022048 for additional details about the system's band structure, localization properties, and scattering matrix invariants.

[38] C. W. Groth, M. Wimmer, A. R. Akhmerov, and X. Waintal, KWANT: A software package for quantum transport, New J. Phys. 16, 063065 (2014).

[39] I. C. Fulga and M. Maksymenko, Scattering matrix invariants of Floquet topological insulators, Phys. Rev. B 93, 075405 (2016).

[40] I. C. Fulga, F. Hassler, and A. R. Akhmerov, Scattering theory of topological insulators and superconductors, Phys. Rev. B 85, 165409 (2012).

[41] E. A. Rodríguez-Mena and L. E. F. Foa Torres, Topological signatures in quantum transport in anomalous Floquet-Anderson insulators, Phys. Rev. B 100, 195429 (2019).

[42] K. W. Kim, D. Bagrets, T. Micklitz, and A. Altland, Quantum Hall criticality in Floquet topological insulators, Phys. Rev. B 101, 165401 (2020).

[43] W. A. Benalcazar, B. A. Bernevig, and T. L. Hughes, Quantized electric multipole insulators, Science 357, 61 (2017).

[44] W. A. Benalcazar, B. A. Bernevig, and T. L. Hughes, Electric multipole moments, topological multipole moment pumping, and chiral hinge states in crystalline insulators, Phys. Rev. B 96, 245115 (2017).

[45] F. Schindler, A. M. Cook, M. G. Vergniory, Z. Wang, S. S. P. Parkin, B. A. Bernevig, and T. Neupert, Higher-order topological insulators, Sci. Adv. 4, eaat0346 (2018).

[46] E. Khalaf, Higher-order topological insulators and superconductors protected by inversion symmetry, Phys. Rev. B 97, 205136 (2018).

[47] L. Trifunovic and P. W. Brouwer, Higher-Order Bulk-Boundary Correspondence for Topological Crystalline Phases, Phys. Rev. X 9, 011012 (2019).

[48] J. M. Edge and J. K. Asbóth, Localization, delocalization, and topological transitions in disordered two-dimensional quantum walks, Phys. Rev. B 91, 104202 (2015).

[49] M. Sajid, J. K. Asbóth, D. Meschede, R. F. Werner, and A. Alberti, Creating anomalous Floquet Chern insulators with magnetic quantum walks, Phys. Rev. B 99, 214303 (2019). 